\title{
Evolution of dwarf spheroidal satellites in the common surface-density dark halos
}

\author{
Yusuke Okayasu ${ }^{1}$ and Masashi Chiba ${ }^{1}$ \\ y.okayasu@astr.tohoku.ac.jp; chiba@astr.tohoku.ac.jp
}

\begin{abstract}
We investigate the growth histories of dark matter halos associated with dwarf satellites in Local Group galaxies and the resultant evolution of the baryonic component. Our model is based on the recently proposed property that the mean surface density of a dark halo inside a radius at maximum circular velocity $V_{\max }$ is universal over a large range of $V_{\max }$. Following that this surface density of $20 M_{\odot} \mathrm{pc}^{-2}$ well explains dwarf satellites in the Milky Way and Andromeda, we find that the evolution of the dark halo in this common surface-density scale is characterized by the rapid increase of the halo mass assembled by the redshift $z_{\mathrm{TT}}$ of the tidal truncation by its host halo, at early epochs of $z_{\mathrm{TT}} \gtrsim 6$ or $V_{\max } \lesssim 22 \mathrm{~km} \mathrm{~s}^{-1}$. This mass growth of the halo is slow at lower $z_{\mathrm{TT}}$ or larger $V_{\max }$. Taking into account the baryon content in this dark halo evolution, under the influence of the ionizing background radiation, we find that the dwarf satellites are divided into roughly two families: those with $V_{\max } \lesssim 22 \mathrm{~km} \mathrm{~s}^{-1}$ having high star formation efficiency and those with larger $V_{\max }$ having less efficient star formation. This semi-analytical model is in agreement with the high-resolution numerical simulation for galaxy formation and with the observed star formation histories for Fornax and Leo II. This suggests that the evolution of a dark halo may play a key role in understanding star formation histories in dwarf satellites.
\end{abstract}

Subject headings: dark matter - galaxies: dwarf - galaxies: formation - galaxies: structure - Local Group

\section{Introduction}

Standard $\Lambda$ cold dark matter $(\Lambda \mathrm{CDM})$ theory is successful for explaining the large-scale structure of the Universe, including the temperature fluctuations of the cosmic microwave background and the spatial distributions of galaxies and their clusters, on scales lager than about $1 \mathrm{Mpc}$ (Tegmark et al. 2004). However, our understanding of structure formation is still incomplete on scales smaller than $1 \mathrm{Mpc}$, i.e. scales of galaxies like our own and their satellite galaxies, which are basic luminous parts of the Universe.

\footnotetext{
${ }^{1}$ Astronomical Institute, Tohoku University, Aoba-ku, Sendai 980-8578, Japan
} 
The tension between the theory and observation on small scales includes the missing satellite problem (Klypin et al. 1999; Moore et al. 1999), too-big-to-fail problem (Bovlan-Kolchin et al. 2011, 2012), and the problems for explaining cored central densities (e.g., Moore et al. 1994; Burkert 1995; de Blok et al. 2001; Swaters et al. 2003; Gilmore et al. 2007; Oh et al. 2011) and anisotropic spatial distribution of dwarf satellites (e.g., Kroupa et al. 2005; McConnachie \& Irwin 2006; Ibata et al. 2013; Pawlowski et al. 2012, 2013, 2015). Solutions to these small-scale issues in $\Lambda$ CDM theory have been considered from two different aspects. One is to modify the standard theory of dark matter on small scales, such as theories based on warm or self-interacting dark matter (e.g., Macciò et al. 2010; Lovell et al. 2012; Vogelsberger et al. 2012). Another way to solve the issues is to rely on the role of baryonic physics in the formation of galaxies and their satellites, including the suppression effects of the ionizing UV background and stellar feedback such as supernova explosion on galaxy formation as well as tidal effects of a centrally concentrated host galaxy on its satellites (e.g., Di Cinto et al. 2014; Madau et al. 2014; Pawlowski et al. 2015; Zhu et al. 2016; Sawala et al. 2016).

In this context, nearby dwarf spheroidal (dSph) galaxies as satellites of the Milky Way and Andromeda galaxies provide ideal sites for studying the nature of dark matter, because stellar dynamics of the resolved member stars suggest that these galaxies are significantly dominated by associated dark matter halos with mass-to-light ratios of 10 to 1000 or more (Gilmore et al. 2007; Simon \& Geha 2007). For this reason, extensive dynamical analyses for these local dSphs have been performed to set constraints on the detailed internal structures of their dark halos and ultimately to get important insights into the nature of dark matter on small scales.

These dynamical analyses of dSphs revealed several universal structures of their dark halos. Mateo et al. (1993) and more recently, Strigari et al. (2008), proposed, based on spherically symmetric mass models, that all the dSphs with luminosities in the range of $10^{3}$ to $10^{7} L_{\odot}$ have a common dark-halo mass of $M_{300} \sim 10^{7} M_{\odot}$ inside an inner 300 pc radius. Following this work, Macciò et al. (2009) showed that this constancy of $M_{300}$ can be naturally explained in the framework of $\Lambda \mathrm{CDM}$ theory combined with galaxy formation physics, where the latter process narrows an allowed range of circular velocities of dark halos. More recently Milosavljevic̀ \& Bromm (2014) showed the growth history of dark halos in this common $M_{300}$ scale and the resultant impacts on the star formation activity in dwarf satellite galaxies.

Other work including Kormendy \& Freeman (2004), Donato et al. (2009) and Gentile et al. (2009) have shown that for the assumed cored isothermal profile or so-called Burkert profile (Burkert 1995) with a density $\rho_{0}$ and radius $r_{0}$ of a core component, the central surface density of a cored dark halo, $\mu_{0 D}=\rho_{0} r_{0}$, is found to be constant, irrespective of the observed $B$-band luminosities of galaxies. The similar universality of a central surface density of a cored dark halo has been proposed by Salucci et al. (2012), arriving at $\mu_{0 D} \simeq 140 M_{\odot} \mathrm{pc}^{-2}$ for both cored isothermal and Burkert profiles. These work indeed provide important constraints on the structure of dark halos and thus on the nature of dark matter. 
Recently, Hayashi \& Chiba (2015a), hereafter referred to as HC15a, proposed a yet another universal scale for the internal structure of dark halos, motivated by the facts that realistic dynamical analysis of non-spherical dSphs based on axisymmetric Jeans equations yields a rather large dispersion in the values of $M_{300}$ (Hayashi \& Chiba 2012) and that the constancy of $\mu_{0 D}$ cannot be applicable to any density profiles including a dark halo with a central cusp. HC15a showed for galaxies spanning the circular velocities of 10 to $400 \mathrm{~km} \mathrm{~s}^{-1}$ that a mean surface density of a dark halo within the radius of the maximum circular velocity, hereafter denoted as $\Sigma_{V_{\max }}$, is remarkably constant, irrespective of the different density distributions in each of the galaxies. It is also shown that this constancy of $\Sigma_{V_{\max }}$ is applied to all the galaxies with $B$-band luminosities over almost 14 orders of magnitude, $M_{B}=-8$ to $-22 \mathrm{mag}$ (Hayashi \& Chiba 2015b), i.e. including faint dSphs to bright spiral/elliptical galaxies.

In this paper, we investigate the evolution of dark matter halos in this common surface-density scale and the resultant impacts on star formation activity in dwarf satellite galaxies, following the work of Milosavljevic̀ \& Bromm (2014) in which the common mass scale of $M_{300}=$ const. for dark halos is assumed. We thus show how the growth histories of dark halos differ between the case of $\Sigma_{V_{\max }}=$ const. and that of $M_{300}=$ const. and how these different assembly histories of dark matter affect the baryonic content and star formation rate in these small-scale dark halos and how the results are compatible with the observed properties of dwarf satellite galaxies in the Milky Way and Andromeda galaxies.

The paper is organized as follows. In Section 2, we calculate the growth histories of dark halos under the constraint of $\Sigma_{V_{\max }}=$ const. and the difference from $M_{300}=$ const.. In Section 3, we consider the baryon content in such dark halos and compare with the remained stellar mass in the currently observed dSphs to estimate the star formation efficiency. In Section 4, to get further insights into the models we develop, we compare with the results of the recent high-resolution numerical simulation, so-called the Illustris simulation, and also compare with the star formation histories of dSphs derived from the recent observations. Section 5 is devoted to our conclusions in this work. In what follows, we adopt the cosmological parameters of a mean matter density $\Omega_{m}=0.2726$, baryon density $\Omega_{b}=0.0456$ and Hubble parameter $h=0.704$.

\section{Structure and evolution of dark halos in dwarf satellites}

\subsection{The common surface-density scale for Local Group dwarf galaxies}

Our mass model for dark halos in Local Group dSphs is assumed to hold a common mean surface density within a radius at maximum circular velocity, $\Sigma_{V_{\max }}=$ const., proposed by HC15a. In the limit of spherical symmetry, $\Sigma_{V_{\max }}$ is given as

$$
\Sigma_{V_{\max }}=\frac{M\left(r_{\max }\right)}{\pi r_{\max }^{2}}
$$


where $r_{\max }$ is a radius at maximum circular velocity (denoted as $V_{\max }$ ) for a given mass profile of a dark halo and $M\left(r_{\max }\right)$ is a mass enclosed within $r_{\max }$,

$$
M\left(r_{\max }\right)=\int_{0}^{r_{\max }} 4 \pi \rho\left(r^{\prime}\right) r^{\prime 2} d r^{\prime}
$$

where $\rho(r)$ denotes a mass density profile of a dark halo. This surface density defined in eq. (10) is basically proportional to the product of a central density, $\rho_{*}$, and a scale length, $r_{*}$, for any density profile of a dark halo, such as the so-called Navarro-Frenk-White (NFW) profile (Navarro et al. 1996) and the Burkert profile (Burkert 1995), where the definition of $\rho_{*}$ and $r_{*}$ depends on a specified form of a given density profile.

HC15a shows that $\Sigma_{V_{\max }}$ is remarkably constant for a large range of $V_{\max }$ from the scales of dwarf to spiral/elliptical galaxies, namely $\Sigma_{V_{\max }}$ is confined within $10^{1} \sim 10^{2} M_{\odot} \mathrm{pc}^{-2}$ over $10 \lesssim V_{\max } \lesssim 400 \mathrm{~km} \mathrm{~s}^{-1}$. This implies that $\Sigma_{V_{\max }}$ is a common surface density scale for the description of a dark halo structure.

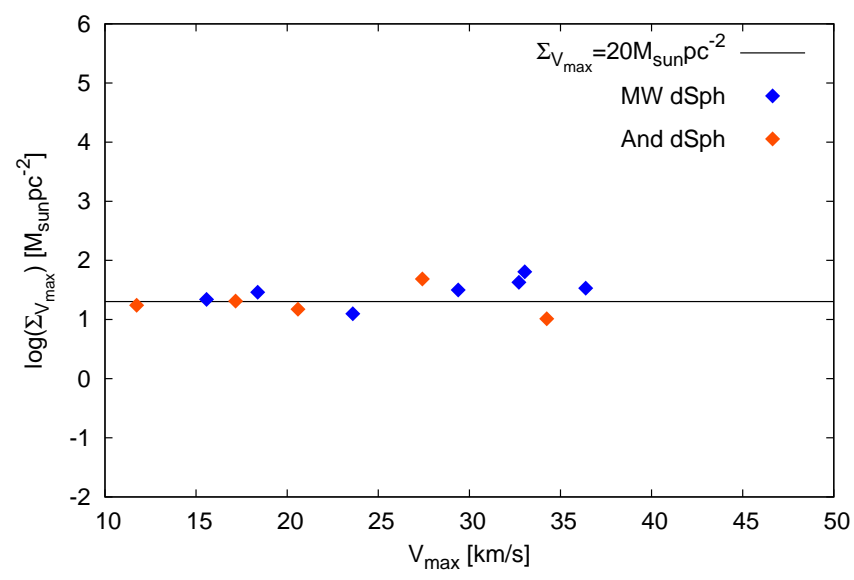

Fig. 1.- Mean surface density of a dark halo, $\Sigma_{V_{\max }}$, within a radius at maximum circular velocity $V_{\max }$, as a function of $V_{\max }$, for the dSphs in the Milky Way (red diamonds) and Andromeda (blue diamonds) taken from HC15a. Horizontal solid line denotes $\Sigma_{V_{\max }}=20 M_{\odot} \mathrm{pc}^{-2}\left(\right.$ or $\log \Sigma_{V_{\max }}=$ $1.3)$.

For our current evolution model of dSphs, we confine ourselves to dwarf satellites in the Milky Way and Andromeda galaxies and adopt the fiducial value of $\Sigma_{V_{\max }}$ for this sample of dSphs. Figure 1 1 shows $\log \Sigma_{V_{\max }}$ vs. $V_{\max }$ for these dSphs taken from the HC15a paper. It is found that $\Sigma_{V_{\max }}=20$ $M_{\odot} \mathrm{pc}^{-2}$ (or $\log \Sigma_{V_{\max }}=1.3$ ) describes the common surface-density scale in these dSphs quite well. In what follows, we use this value of $\Sigma_{V_{\max }}$ as a fiducial common surface-density scale to describe the evolution of dark halos in dwarf satellites, and further consider neighboring values of $\Sigma_{V_{\max }}=10$ $M_{\odot} \mathrm{pc}^{-2}$ (or $\left.\log \Sigma_{V_{\max }}=1.0\right)$ and $40 M_{\odot} \mathrm{pc}^{-2}$ (or $\log \Sigma_{V_{\max }}=1.6$ ) to investigate the dependence of the following results on the variation of $\Sigma_{V_{\max }}$. 


\subsection{Evolution of the NFW dark halos}

To obtain the evolution of dark halos under the constraint that $\Sigma_{V_{\max }} \simeq 20 M_{\odot} \mathrm{pc}^{-2}$, we here assume that the halo density profile follows the NFW profile with a central density $\rho_{s}$ and a scale length $r_{s}$,

$$
\left.\rho_{(} r\right)=\frac{\rho_{s}}{\left(r / r_{s}\right)\left(1+r / r_{s}\right)^{2}} \text {. }
$$

For this density profile, $V_{\max }$ is attained at $r_{\max }=2.16 r_{s}$ and $\Sigma_{V_{\max }}$ is then exactly proportional to $\rho_{s} r_{s}$. This NFW profile is parametrized by a concentration parameter $c=r_{\text {vir }} / r_{s}$, where $r_{\text {vir }}$ is the virial radius defined such that the mean density inside $r_{\text {vir }}$ equals 200 times the critical density of the Universe. We use the calibration of the halo virial mass, $M_{\text {vir }}$, and redshift dependence of the median halo concentration, $c\left(M_{\mathrm{vir}}, z\right)$, derived by Prada et al. (2012) for halos in the Bolshoi and MultiDark simulations, as adopted in Milosavljevic̀ \& Bromm (2014).
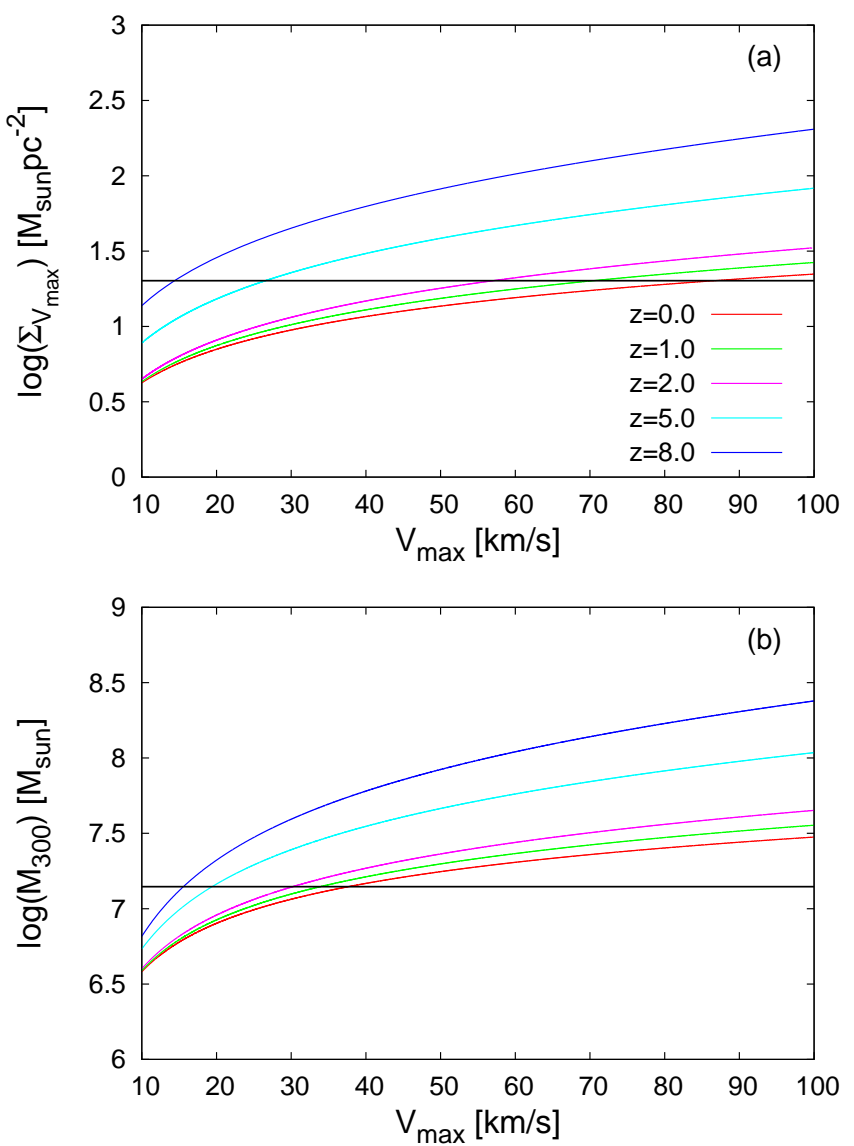

Fig. 2.- (a) Redshift evolution of $\Sigma_{V_{\max }}$ vs. $V_{\max }$ at $z=0$ for the NFW dark halo model. Horizontal line denotes $\Sigma_{V_{\max }}=20 M_{\odot} \mathrm{pc}^{-2}$ (or $\log \Sigma_{V_{\max }}=1.3$ ). (b) Redshift evolution of $M_{300}$ vs. $V_{\max }$ at $z=0$ for the NFW dark halo model. Horizontal line denotes $M_{300}=1.4 \times 10^{7} M_{\odot}$ obtained for classical dwarfs. 
Figure 2(a) shows $\Sigma_{V_{\max }}$ vs. $V_{\max }$ at $z=0$ for this NFW dark halo model. As is clear from this figure, a mean surface density inside $r_{\max }$ shows only a weak dependence on the current maximum circular velocity or total mass of a dark halo. This reflects that such inner regions of all the halos having different total masses formed basically from very early mergers of small and dense halos and these high density regions have been rather undisturbed by later accretion of halos. Nonetheless, the mean surface density of halos is not exactly constant for different mass but varies, depending on redshifts, in comparison with the observed constancy of $\Sigma_{V_{\max }}=20 M_{\odot} \mathrm{pc}^{-2}$ (horizontal black line).

This property of a halo is also seen when we adopt an inner mass of a dark halo instead of an inner surface density as a common scale; Figure 2(b) shows an inner mass, $M_{300}$, enclosed within $r=300$ pc (Strigari et al. 2008) as a function of $V_{\max }$. As already demonstrated in Macciò et al. (2009) (see also Kravtsov 2010), $M_{300}$ also shows a weak dependence on $V_{\max }$ of a halo for the same reason given for $\Sigma_{V_{\max }}$. In this panel, the horizontal black line corresponds to $M_{300}=1.4 \times 10^{7}$ $M_{\odot}$, which is a likely common value when we adopt the results of Jeans analysis only for bright, classical dwarfs under the assumption of spherical symmetry (Strigari et al. 2008); including ultrafaint dSphs (UFDs) suggests a somewhat smaller common scale of $M_{300} \simeq 10^{7} M_{\odot}$, but UFDs generally show a large deviation from spherical symmetry and thus this latter value needs to be taken in caution.

Comparing both panels in Fig. 2, it follows that the common surface density scale, $\Sigma_{V_{\max }}=20$ $M_{\odot} \mathrm{pc}^{-2}$, covers quite a large range of $V_{\max }$ at different redshifts, whereas $M_{300}=1.4 \times 10^{7} M_{\odot}$ is applicable only for $V_{\max } \lesssim 40 \mathrm{~km} \mathrm{~s}^{-1}$. This suggests that the use of $\Sigma_{V_{\max }}$ may be more general in comparison with $M_{300}$ as a common scale for the evolution of a dark halo.

\subsection{Basic properties of dark halos with the common surface-density scale}

To understand the constancy of $\Sigma_{V_{\max }}$ at redshift $z=0$ and that of $M_{300}$ as well, we need to consider the termination of mass growth in these dark halos associated with satellite galaxies, when they are accreted into a host halo, i.e. the halo of the Milky Way or Andromeda, due to tidal force. Following the model of Milosavljević \& Bromm (2014), we assume that the halos have a tidally truncated maximum mass, $M_{\mathrm{TT}}$, when they are accreted into a host halo at redshift $z_{\mathrm{TT}}$. Then, we obtain a family of a dark halo with $M_{\mathrm{TT}}\left(z_{\mathrm{TT}}\right)$ so as to satisfy the common surface-density scale, $\Sigma_{V_{\max }}=20 M_{\odot} \mathrm{pc}^{-2}$.

The top panel in Fig. 3 shows $1+z_{\mathrm{TT}}$ vs. $M_{\mathrm{TT}}$ under this common surface-density scale (solid line) in comparison with the common $M_{300}$ scale (dashed line). We also plot the cases of $\Sigma_{V_{\max }}=40 M_{\odot} \mathrm{pc}^{-2}$ (dot-dashed line) and $10 M_{\odot} \mathrm{pc}^{-2}$ (dotted line) for comparison. For the common surface-density scale of $\Sigma_{V_{\max }}=20 M_{\odot} \mathrm{pc}^{-2}$, we obtain solutions with non-negative tidal truncation redshifts for the mass range of $M_{\mathrm{TT}}<2 \times 10^{10} M_{\odot}$, which is an order of magnitude wider than the case of the common $M_{300}$ scale $\left(M_{\mathrm{TT}}<1.2 \times 10^{9} M_{\odot}\right)$. This result reflects the property 


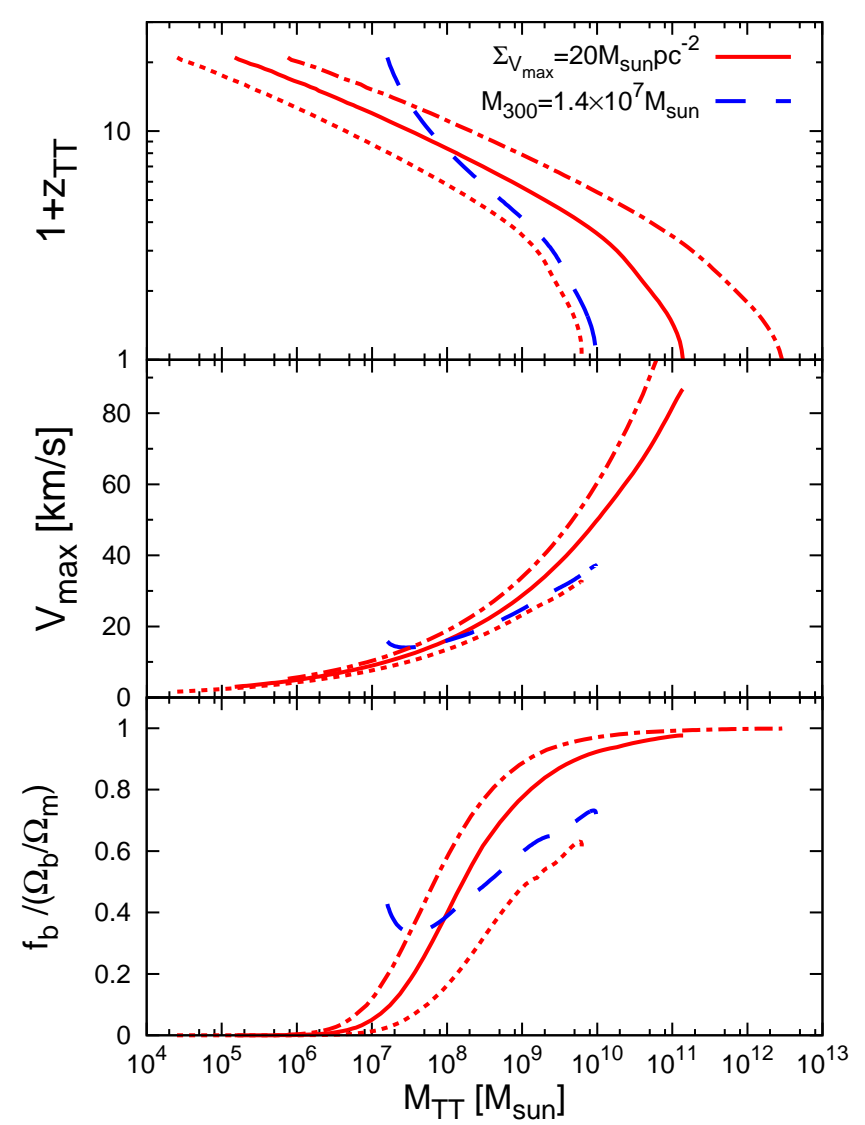

Fig. 3.- Properties of the dark halos with mass $M_{\mathrm{TT}}$ having the common surface-density scale of $\Sigma_{V_{\max }}=20 M_{\odot} \mathrm{pc}^{-2}$ (solid line), compared with the case for the common mass scale of $M_{300}=$ $1.4 \times 10^{7} M_{\odot}$ (dashed line). The dot-dashed and dotted lines show, respectively, the cases of $\Sigma_{V_{\max }}=40 M_{\odot} \mathrm{pc}^{-2}$ and $10 M_{\odot} \mathrm{pc}^{-2}$. Top panel: redshift $z_{\mathrm{TT}}$ vs. $M_{\mathrm{TT}}$. Middle panel: maximum circular velocity $V_{\max }$ vs. $M_{\mathrm{TT}}$. Bottom panel: baryon fraction in units of the universal baryon fraction $f_{b}\left(\Omega_{b} / \Omega_{m}\right)^{-1}$ vs. $M_{\mathrm{TT}}$. 
shown in Fig. 2 that $\Sigma_{V_{\max }}=20 M_{\odot} \mathrm{pc}^{-2}$ covers a larger range of $V_{\max }$ than $M_{300}=1.4 \times 10^{7} M_{\odot}$. While the increase of $M_{\mathrm{TT}}$ with decreasing $z_{\mathrm{TT}}$ is gradual at $z_{\mathrm{TT}} \leq 2$ on both scales, the slope is steeper at higher $z_{\mathrm{TT}}$, where the case of the common $\Sigma_{V_{\max }}$ scale provides a more rapid increase of $M_{\mathrm{TT}}$ with decreasing $z_{\mathrm{TT}}$ than the common $M_{300}$ scale. These properties of dark halo evolution are also seen in Fig 2, where $z=$ const. lines are less dense at $z>2$, especially for the evolution of $\Sigma_{V_{\max }}$ at smaller $V_{\max }$. This suggests that there is a systematic difference in the mass growth of a dark halo at high redshifts between the cases of the common $\Sigma_{V_{\max }}$ and $M_{300}$ scales. We note that adopting the different value of $\Sigma_{V_{\max }}=10$ or $40 M_{\odot} \mathrm{pc}^{-2}$ leads to the change of an upper limit for $M_{\mathrm{TT}}$ by an order of magnitude but the general dependence of $1+z_{\mathrm{TT}}$ on $M_{\mathrm{TT}}$ remains basically the same.

The middle panel in Fig. 3 shows $V_{\max }$ for these common scales. As already mentioned in Fig. 2, a dark halo with $\Sigma_{V_{\max }}=20 M_{\odot} \mathrm{pc}^{-2}$ covers the large mass range of $10^{5} M_{\odot}$ to $10^{11} M_{\odot}$, so that the associated range for $V_{\max }$ is $5 \lesssim V_{\max } \lesssim 90 \mathrm{~km} \mathrm{~s}^{-1}$, namely including both the smalland large-mass galaxies. This is in contrast to the common $M_{300}$ scale, where the applicable range of $V_{\max }$ is limited. This limitation for the range of $V_{\max }$ is also seen when we adopt $\Sigma_{V_{\max }}=10$ $M_{\odot} \mathrm{pc}^{-2}$ : only $V_{\max } \lesssim 32 \mathrm{~km} \mathrm{~s}^{-1}$ is allowed, which can also be deduced from Fig. 2(a).

\section{Evolution of the baryonic component}

\subsection{Baryonic content}

Formation of dSphs is intimately affected by the cosmic reionization, whereby gas inflow into dark-matter subhalos associated with dSphs is inhibited and/or gas is flowing out of a gravitational potential due to photoionization. To what extent baryon is actually retained in each of subhalos is thus dependent on the evolution of photo-ionized gas in the presence of the ionizing background radiation, given the redshift evolution of dark halo density profiles on the scale of dSphs. This baryonic content is quantified by the baryon fraction $f_{b}$ in each mass of dark halos in units of universal baryonic fraction $\Omega_{b} / \Omega_{m}$, where $\Omega_{b}$ and $\Omega_{m}$ denote cosmic densities of baryon and matter normalized by a critical density of the Universe, respectively.

In this work, for the purpose of highlighting the effects of adopting different evolutionary histories of dark halos on the estimate of $f_{b}$, we follow the prescription for formulating it by Milosavljević \& Bromm (2014), using the critical halo mass, $M_{\text {crit }}$, for retaining the half of universal baryon mass, defined as $f_{b}\left(M_{\text {crit }}, z\right)=\Omega_{b} / 2 \Omega_{m}$. We adopt $M_{\text {crit }}(z)$ as given in Milosavlievic̀ \& Bromm (2014)

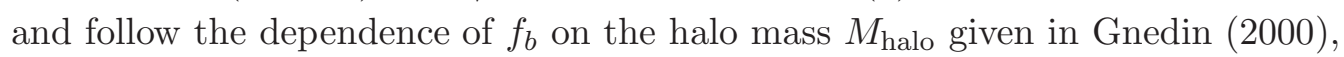

$$
f_{b}\left(M_{\text {halo }}, z\right)=\frac{\Omega_{b}}{\Omega_{m}}\left(1+\left(2^{\alpha / 3}-1\right)\left[\frac{M_{\text {halo }}}{M_{\text {crit }}(z)}\right]^{-\alpha}\right)^{-3 / \alpha},
$$

where $\alpha$ is a shape parameter, for which we adopt $\alpha=1$ (Gnedin 2012; Sobacchi \& Mesinger 2013) (see also, Okamoto et al. 2008). For the $z=0$ value of $M_{\text {crit }}(z)$, we adopt $M_{\text {crit }}(0)=7 \times 10^{9} M_{\odot}$ 
(Okamoto et al. 2008) as in Milosavljevic̀ \& Bromm (2014).

The bottom panel in Fig. 3 shows the baryon fraction in units of $\Omega_{b} / \Omega_{m}$ at the epoch of the tidal truncation of mass assembly. It is found that for the low mass range of dark halos formed at high $z_{\mathrm{TT}}$. their virial temperatures are too low to retain baryonic gas, i.e., $f_{b}=0$, on such low-mass scales. At lower redshifts of $z_{\mathrm{TT}} \lesssim 10, f_{b}$ is increasing with $M_{\mathrm{TT}}$ and tending to the universal baryon fraction at the high-mass end. On the contrary, for the case of the common $M_{300}$ scale, since dark halos are already massive enough at high $z_{\mathrm{TT}}$ to retain baryon and their masses grow only gradually with decreasing $z_{\mathrm{TT}}$ as shown in the top panel of Fig. 3, the dependence of $f_{b}$ on $M_{\mathrm{TT}}$ is somewhat weaker than the case of the common $\Sigma_{V_{\max }}$ scale. This difference in the dependence of the baryon fraction on $M_{\mathrm{TT}}$ between these common scales results in the different implications for star formation activity in dwarf satellites as explained below.

\subsection{Comparison with observed dwarf satellites}

The estimated value of the baryon content $f_{b}$ at the epoch of the tidal truncation of mass assembly is likely a maximum amount of available gas to form stars in each of dwarf satellites. Then, during the resultant course of orbital motions within a gravitational potential of a host halo, gas in each satellite may be lost in the form of outflow driven by supernova feedback or eventually completely removed by ram pressure stripping, at which star formation is finally quenched.

We estimate how much gas has actually been converted into stars in each of dwarf satellites using the observationally determined stellar mass and then derive how much gas has been eventually lost from each system. We define

$$
F=\frac{M_{\text {star }}}{M_{\text {baryon }}}
$$

where $M_{\text {star }}$ denotes the total mass of stars observed at the current epoch and $M_{\text {baryon }}$ is the available gas mass (estimated from $f_{b}$ at the epoch of the tidal truncation of mass assembly) in each of satellite galaxies. $F$ is thus a measure of star formation efficiency, where $F=1$ indicates that all the gas has been converted into stars and $F<1$ implies the loss of gas from the system by external effects such as ram-pressure and/or tidal stripping process or internal effects such as heating of gas from supernova feedback. Thus, $1-F$ tells us the total amount of gas lost from the system, thereby providing important information for understanding star formation and chemical evolution histories in dwarf galaxies.

Figure 4 shows $M_{\text {star }}$ vs. $V_{\max }$ for dSphs in the Milky Way and Andromeda galaxies. The mass of stars in each dSph is based on the estimate of the mass-to-luminosity ratios in the $V$ band magnitude, $M_{\mathrm{star}} / L_{\mathrm{V}}$, by Woo et al. (2008), where the values of $L_{\mathrm{V}}$ for these sample galaxies are taken from the data assembled and listed in Kirby et al. (2014). Their mean metallicities are shown in terms of the size of each mark, for which the data are taken from Kirby et al. (2013). Solid lines show our semi-analytical model for constant $F$ sequences, in the fiducial case of $\Sigma_{V_{\max }}=20$ 


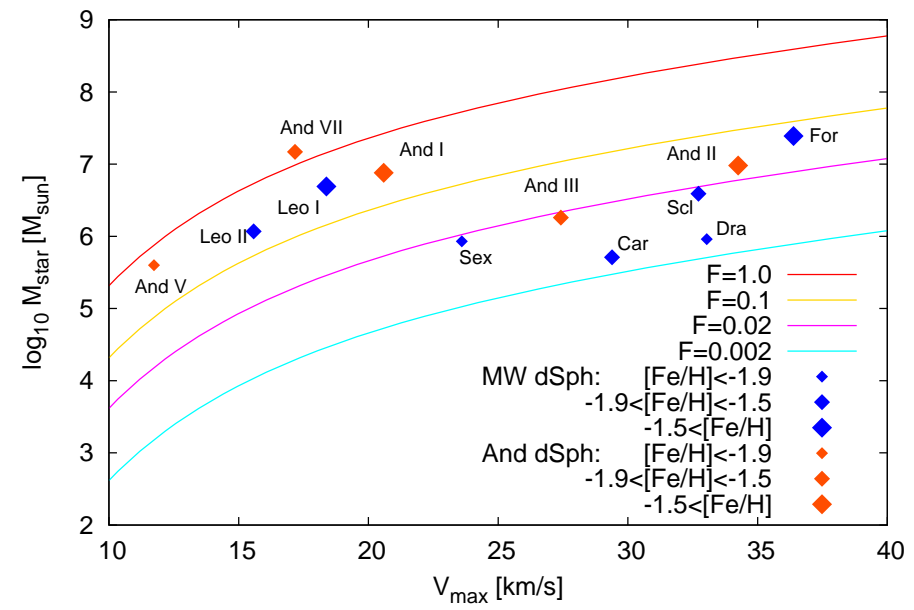

Fig. 4.- Stellar mass $M_{\text {star }}$ vs. $V_{\max }$ for the dSphs in the Milky Way (red diamonds) and Andromeda (blue diamonds), with larger sizes of the marks for higher metallicities. Solid lines shows, from top to bottom, the semi-analytical model for $F=1.0,0.1,0.02$ and 0.002 in the fiducial case of $\Sigma_{V_{\max }}=20 M_{\odot} \mathrm{pc}^{-2}$.

$M_{\odot} \mathrm{pc}^{-2}$. It follows from the figure that the current sample of dwarf satellites is divided into roughly two different families of $F>0.1$ at $V_{\max } \lesssim 22 \mathrm{~km} \mathrm{~s}^{-1}$ (And V, Leo II, And VII, Leo I and And I) and $F<0.1$ at $V_{\max } \gtrsim 22 \mathrm{~km} \mathrm{~s}^{-1}$ (Sextans, And III, Carina, Sculptor, Draco, And II and Fornax). The former, low mass dSphs, are characterized by $z_{\mathrm{TT}} \gtrsim 6$, at which stars formed efficiently before the effect of the reionization became significant, whereas star formation efficiency in the latter, more massive dSphs may be reduced because of the presence of the ionizing background by the time they are accreted into a host halo. It is also suggested that except for And VII, all the dSphs imply $F<0.3$ and thus more than $70 \%$ of gas is lost from the system. This is indeed supported by recent chemical evolution models (e.g., Homma et al. 2015) to understand the observed metal-poor distributions of stars in dSphs.

For And VII, we obtain $F=1.45$ with $M_{\text {baryon }}=1.02 \times 10^{7} M_{\odot}$ at $z_{\mathrm{TT}}=7.06$ and $M_{\text {star }}=$ $1.48 \times 10^{7} M_{\odot}$, suggesting quite an efficient conversion of gas into stars, although $M_{\text {star }}>M_{\text {baryon }}$ at the redshift of the tidal truncation needs explanations. This is consistent with the Hubble Space Telescope results by Weisz et al. (2014) that more than $90 \%$ of the stars in And VII formed within the first 1 Gyr. Regarding $F>1$ in this galaxy, it is interesting to note that the distance from the Andromeda center to this satellite is largest $(218 \mathrm{kpc})$ among the current sample of the Andromeda satellites, whereby this galaxy may be allowed to obtain the further gas infall after the epoch of $z_{\mathrm{TT}}$ and form extra stars before the ram pressure stripping removes gas completely.

Figure 5 shows the effects of adopting neighboring values of $\Sigma_{V_{\max }}=10 \mathrm{M}_{\odot} \mathrm{pc}^{-2}$ (a) and 40 $M_{\odot} \mathrm{pc}^{-2}(\mathrm{~b})$. It follows that the general properties of constant $F$ lines relative to observed dSphs remain basically the same as the fiducial case of $\Sigma_{V_{\max }}=20 M_{\odot} \mathrm{pc}^{-2}$, namely the dSphs can be divided into those with high or low $F$, depending on the value of $V_{\max }$. In addition, compared with 
the fiducial case of $\Sigma_{V_{\max }}=20 M_{\odot} \mathrm{pc}^{-2}$, adopting a smaller (larger) $\Sigma_{V_{\max }}$ results in a smaller (larger) $F$, thereby implying that dSphs having higher surface densities at a given $V_{\max }$ are expected to show higher star formation efficiency and thus may cause some dispersion in the distribution of F.
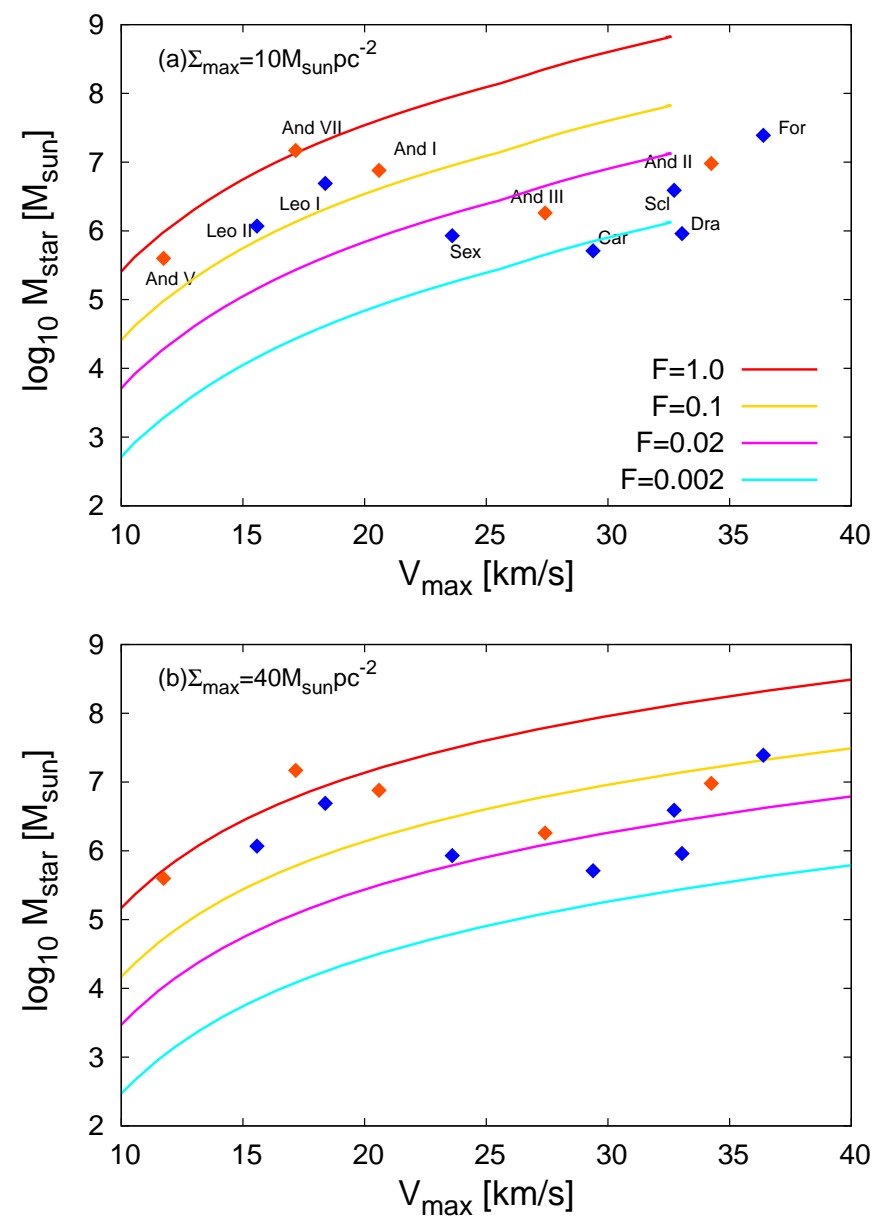

Fig. 5.- The same as Fig. 4, but for the different common surface-density scales of $\Sigma_{V_{\max }}=10$ $M_{\odot} \mathrm{pc}^{-2}$ (a) and $40 M_{\odot} \mathrm{pc}^{-2}(\mathrm{~b})$. For the former case, solutions are available only for $V_{\max } \leq 32$ $\mathrm{km} \mathrm{s}^{-1}$, as shown in the middle panel of Fig. 3.

We note that in the Milosavljevic̀ \& Bromm (2014) paper adopting $M_{300}=$ const., halos with high baryon fraction are thought to form stars in isolated/nuclear clusters, whereas halos with low baryon fraction imply low star formation efficiency and hence galaxies are expected to stay dark. Thus, the adoption of a different common scale leads to a different interpretation for star formation activity in dSphs. 


\section{Discussion}

\subsection{Comparison with the high-resolution numerical simulation}

Our model of dwarf galaxies in the common surface-density scale combined with gas physics in the ionizing background suggests that there are broadly two families of satellite galaxies, those experiencing efficient star formation with $F>0.1$ at early epochs and those with inefficient star formation with $F<0.1$ due to the effects of the ionizing background at lower redshifts. These different types of satellite galaxies may be divided at $V_{\max }$ of about $22 \mathrm{~km} \mathrm{~s}^{-1}$. However, the number of sample dSphs in the Milky Way and Andromeda galaxies for the current analysis is limited only to 11 at a moment, so it is yet unclear whether this suggested property is indeed the case.

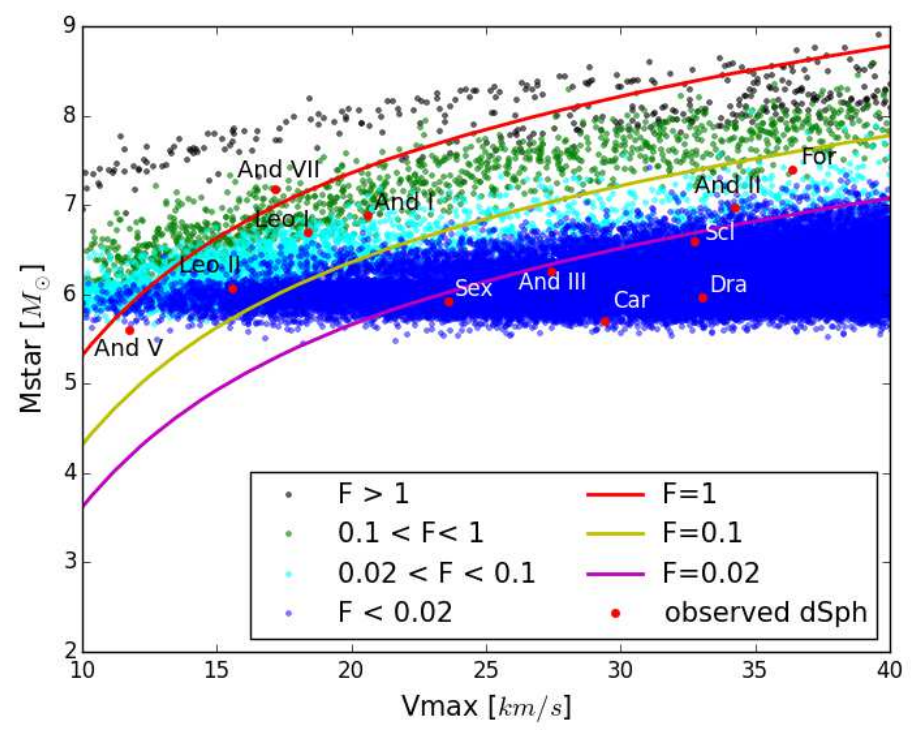

Fig. 6.- $M_{\text {star }}$ vs. $V_{\max }$ for subhalos in the Illustris simulation (dots). Simulation results are divided into 4 different ranges in $F$. Solid lines correspond to our semi-analytical models for $F$ and observed data are shown with red circles.

To remedy this situation, we investigate the results of the recent high-resolution numerical simulation for galaxy formation in the fully cosmological context, where the reionization of the Universe is taken into account. For this purpose, we adopt the Illustris simulation (Nelson et al. 2015). We derive $F$ defined in eq. (5) associated with each dark-matter subhalo at $z=0$ given in the Illustris data, where $M_{\text {baryon }}$ is estimated by multiplying the mass of each subhalo by the universal baryon fraction of $\Omega_{b} / \Omega_{m}$. Note that in this procedure of deriving $M_{\text {baryon }}$ from the simulation results, a finite fraction of subhalos may have continued their growth up to $z=0$, in contrast to our semi-analytical model assuming the tidal truncation at $z_{\mathrm{TT}}$. This suggests that we may overestimate $M_{\text {baryon }}$ and thus underestimate $F$ from the simulation results to some extent, compared with those in our semi-analytical model. 
Figure [ shows the $M_{\text {star }}$ vs. $V_{\max }$ for the simulated subhalos, which are divided into 4 different ranges in $F$. Solid lines correspond to our semi-analytical models for $F$ and observed data are shown with red circles. Although there exists some discrepancy between our model and numerical simulation results at $V_{\max }<20 \mathrm{~km} \mathrm{~s}^{-1}$, which may be partially due to insufficient resolution in numerical simulation on such small scales or other effects such as later gas infall into subhalo after the tidal truncation by their host halo, it is quite impressive that a very good agreement is achieved at $V_{\max }>25 \mathrm{~km} \mathrm{~s}^{-1}$, including the slope of a constant $F$ line in the diagram, although we never make any fine tuning in our model.

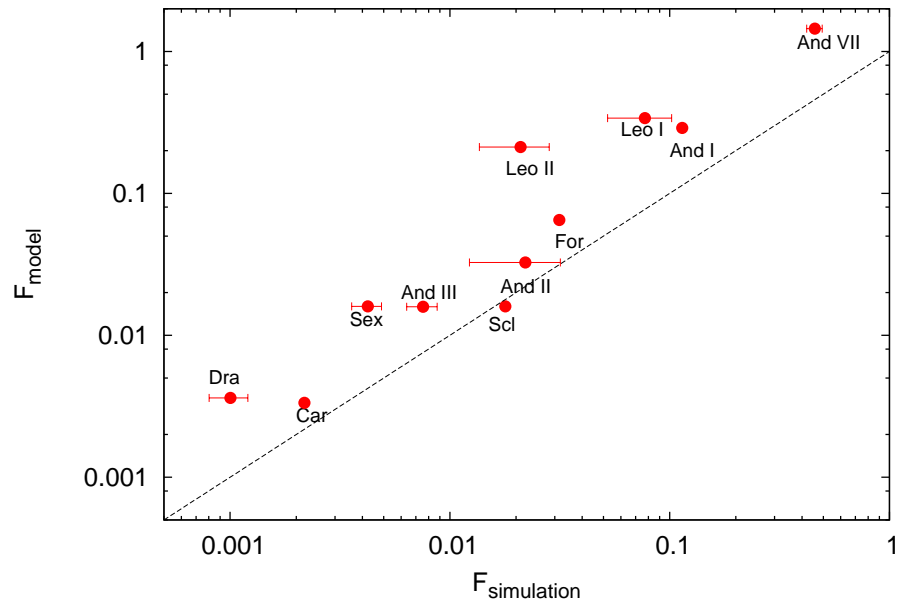

Fig. 7.- Comparison between $F$ obtained from the Illustris simulation and that from our semianalytical model.

Indeed, Fig. 7 shows the comparison between $F$ obtained from the Illustris simulation and that from our semi-analytical model. This figure clearly demonstrates that there is a very reasonable agreement between these values of $F$, although $F$ from the simulation results appears slightly smaller than that in the semi-analytical model due to the reason mentioned above. This may support our hypothesis that there exist roughly two different families of dwarf galaxies in terms of star formation efficiency, which is probably controlled by external and/or internal feedback effects including the ionizing background radiation and/or supernova explosions.

With this $F$ value, it is also possible to estimate the integrated amount of mass loss from dwarf satellites in terms of $1-F$, which may ultimately affect their chemical evolution histories. To infer this property in the Illustris simulation data, we plot, in Fig. 8, $1-F$ vs. the mass-weighted average metallicity of the star particles $M_{\mathrm{Z} \text {,star }} / M_{\text {tot,star }}$ in each subhalo, for different ranges of $V_{\max }$. In this plot, to avoid the effect of the mass-metallicity degeneracy in the result, we adopt only the subhalos having $M_{\text {tot,star }}=(1.00 \pm 0.05) \times 10^{7} M_{\odot}$. Although there are large dispersions in the plot, it follows that a subhalo with larger $1-F$ tends to have a more metal-poor stellar system. The solid line corresponds to a fiducial chemical evolution model including a gaseous outflow, where the outflow rate is assumed to be proportional to the star formation rate and the 
resultant effective yield is taken as 0.7 . Although we do not intend to fit this line to the simulation data, the model reasonably reproduces the properties of subhalos especially with $V_{\max }$ larger than $\sim 30 \mathrm{~km} \mathrm{~s}^{-1}$, thereby implying that a subhalo losing more gas and metals as well leaves a more metal-poor dwarf galaxy. We note that for subhalos with smaller $V_{\max }$, the simulation data show systematically more metal-poor stars for given $1-F$ than the case of the solid line. Physically this may imply the loss of metals independent of the loss of gas in such subhalos, e.g. by means of the metal-enhanced supernova wind (Mac Low \& Ferrara 1999; Martin et al. 2002; Kirby et al. 2011a,b; McQuinn et al. 2015). Alternatively, this may be just an artifact of rather low numerical resolution of Illustris at $V_{\max }<25 \mathrm{~km} \mathrm{~s}^{-1}$, where as shown in Fig. 6, our semi-analytical model for a constant $F$ differs from $F$ deduced from Illustris: the latter predicts a systematically smaller $F$ than our model and thus lower star formation efficiency, so that a stellar system is made more metal-poor than the case for larger $V_{\max }$. Further investigation for this issue is needed, based on much higher-resolu ${ }^{\perp}$

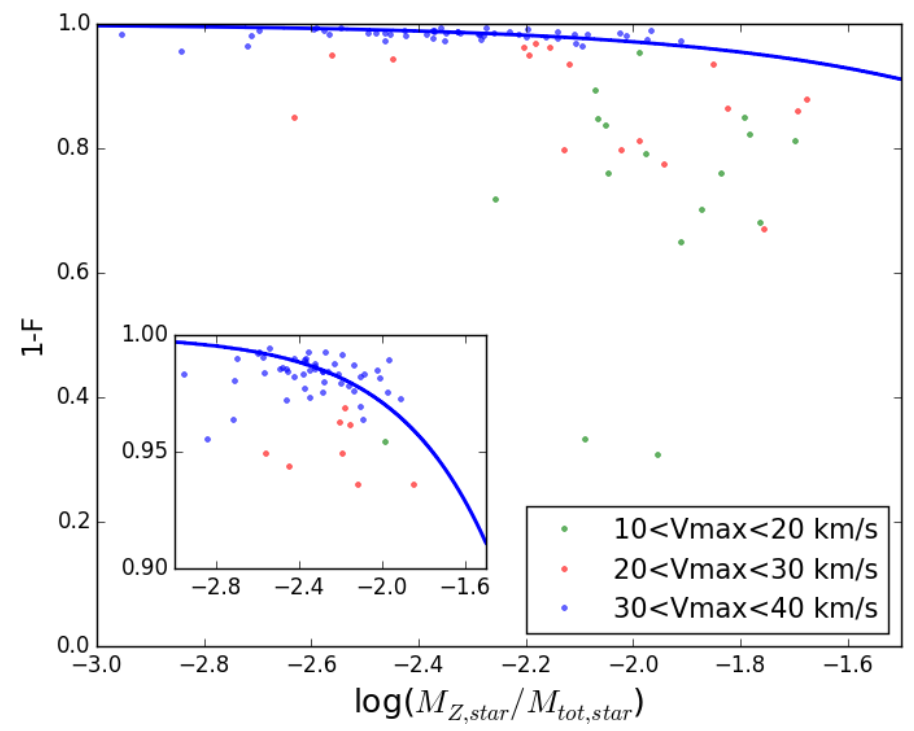

Fig. 8.- Comparison between $1-F$ and the stellar metallicity in each subhalo for the Illustris simulation, where the latter is estimated from the mass-weighted average metallicity of the star particles $M_{\mathrm{Z}, \mathrm{star}} / M_{\mathrm{tot}, \mathrm{star}}$. In this plot, to avoid the effect of the mass-metallicity degeneracy, we adopt only the subhalos having $M_{\text {tot,star }}=(1.00 \pm 0.05) \times 10^{7} M_{\odot}$. Solid line denotes the prediction of a fiducial chemical evolution model including a gaseous outflow, where the outflow rate is assumed to be proportional to the star formation rate and we adopt the resultant effective yield as 0.7 . The inset shows the plots in the range of $0.9 \leq 1-F \leq 1.0$ for clarity. 


\subsection{Star formation history}

Dark halos in dwarf satellite galaxies continue to grow via mass accretion from outside until they enter a host halo at $z_{\mathrm{TT}}$ after which the resultant tidal stripping inhibits a further mass growth. The ram-pressure stripping may also be at work to prevent further gas infall and thus star formation. Thus we expect that the main star formation activity in each of dwarf galaxies occurs prior to $z_{\mathrm{TT}}$, where the continuous growth of dark halos accompanies gas inflow simultaneously, from which new stars are formed.

Following this conjecture, we calculate the inflow rate of gas from outside, based on the associated mass growth in a dark halo component. For this purpose, we adopt the growth history of a dark halo, $d \ln M_{\text {halo }} / d z$, given in equation (7) of the Milosavljevic̀ \& Bromm (2014) paper:

$$
\frac{d \ln M_{\text {halo }}}{d z}=-0.62\left(\frac{1+1.11 z}{1+z}\right)\left[\frac{d \sigma^{2} / d \ln M\left(M_{\text {halo }}\right)}{d \sigma^{2} / d \ln M\left(10^{12} M_{\odot}\right)}\right]^{-1 / 2},
$$

where $\sigma(M)$ denotes the rms fluctuation of the density field linearly extrapolated to $z=0$. This equation corresponds to the growth rate of the mean most massive progenitor for halos, which now belong to the common surface-density scale family in this work. We assume that the inflow rate of gas is simply given as the baryon fraction times the growth rate of a dark halo, i.e., being proportional to $d M_{\text {halo }} / d t$.
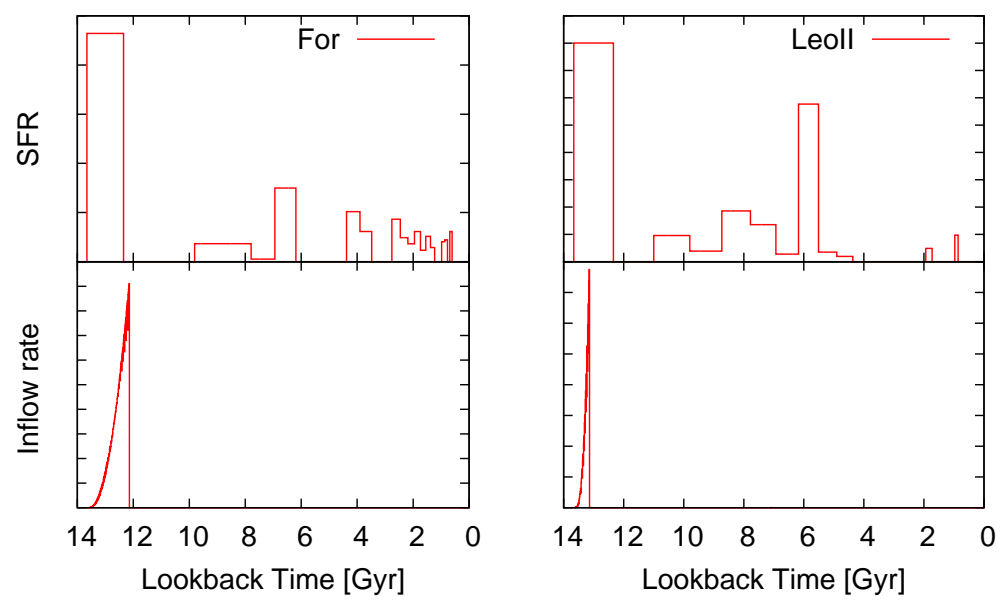

Fig. 9.- Upper panels: SFR at each epoch calculated from the observed cumulative SFR (Weisz et al. 2014) for Fornax (left) and Leo II (right). Lower panels: Gaseous infall rate based on our dark-halo model in the common surface-density scale for Fornax (left) and Leo II (right). In both upper and lower panels, the vertical axis is arbitrarily normalized.

In this work, we choose Fornax and Leo II as two representative cases, where the epochs of tidal truncation under a constraint of the common surface-density scale of $20 M_{\odot} \mathrm{pc}^{-2}$ are given as $z_{\mathrm{TT}}=3.75$ and 7.57 , respectively, i.e. subsequent and prior to the completion of the cosmic reionization. For these dSphs, the values of $F$ indicating star formation efficiency are estimated as 
0.065 (Fornax) and 0.212 (Leo II), thereby suggesting that the former dSph with $z_{\mathrm{TT}}$ being lower than the redshift for the completion of the cosmic reionization is subject to the suppression of star formation caused by the ionizing background radiation.

To estimate the star formation histories in these dSphs, we adopt the Hubble Space Telescope results by Weisz et al. (2014) for the Milky Way dSphs, based on the distribution of resolved member stars in the color-magnitude diagram. Since in the Weisz et al. (2014) paper, they showed the cumulative star formation rate (SFR), we evaluate the SFR at each epoch by differentiating the cumulative SFR. Upper panels in Fig. 9] show the (normalized) SFR at each epoch for Fornax (upper left) and Leo II (upper right). Lower panels in this figure show our model for the (normalized) infall rate of gas based on the dark matter growth in the common surface-density scale, for Fornax (lower left) and Leo II (lower right). In these lower panels, the inflow rate of gas is maximum at redshift $z_{\mathrm{TT}}$, i.e. at the epoch of tidal truncation when a subhalo enters into a host halo at the corresponding epoch. Figure 9 suggests that the observed peak of the star formation rate in Fornax and Leo II is indeed in good agreement with the epoch when the peak of the gas infall rate is achieved. This implies that star formation activity in these dwarf satellites may be controlled by the mass growth in a dark halo component under a constraint of the common surface-density scale of $\Sigma_{V_{\max }} \simeq 20 M_{\odot} \mathrm{pc}^{-2}$ yielding $z_{\mathrm{TT}}$, i.e., the peak epoch of the gas infall rate, and this epoch is to be compared with the completion epoch of the cosmic reionization to infer the effect on star formation efficiency. Further tests for the current semi-analytical model are needed, in which much tighter limits on star formation histories of these galaxies, e.g. based on measurements of detailed abundance patterns of member stars, may enable us to deduce any differences in star formation efficiency between the two families of dSphs.

\section{Conclusions}

We have investigated the mass growth of dark halos in dwarf galaxy satellites in Local Group galaxies, under the recently proposed property by HC15a that the mean surface density of a dark halo inside a radius at maximum circular velocity is universal over a large range of luminosities and masses of galaxies. Following that this surface density $\Sigma_{V_{\max }}$ with $20 M_{\odot} \mathrm{pc}^{-2}$ well explains dwarf satellite galaxies in the Milky Way and Andromeda galaxies, we calculate the evolution of dark halos and associated evolutionary histories of the baryonic component in dwarf satellites. Our main results are summarized as follows.

- Dark halos in the common surface-density scale show the rapid mass growth at high redshifts, while they evolve only slowly at lower redshifts of $z_{\mathrm{TT}}<2$. It is found that these dark halos cover a large range of $V_{\max }$ including the scales of UFDs with $V_{\max }$ as small as $10 \mathrm{~km} \mathrm{~s}^{-1}$ as well as those of bright galaxies with $V_{\max }$ as large as a few hundred $\mathrm{km} \mathrm{s}^{-1}$. This is in contrast to dark halos in the common mass scale of $M_{300} \simeq 1.4 \times 10^{7} M_{\odot} \mathrm{pc}^{-2}$, which covers only a limited range of $V_{\max }$. Thus, the mean surface density $\Sigma_{V_{\max }}$ provides a more general 
description for the evolution of dark halos.

- The calculation of the baryon fraction retained in these dark halos in the presence of the ionizing background radiation reveals that low-mass satellites with $V_{\text {max }}$ below $\sim 22 \mathrm{~km} \mathrm{~s}^{-1}$ are characterized by high star formation efficiency of $F>0.1$, whereas more massive satellites with $V_{\text {max }}$ being larger than $\sim 22 \mathrm{~km} \mathrm{~s}^{-1}$ show low star formation efficiency of $F<0.1$. This is understood on the basis of the mass growth of dark halos and its termination at $z_{\mathrm{TT}}$ in the following manner. Low $V_{\max }$ halos grow fast at high redshifts and are less affected by the ionizing radiation, whereby star formation efficiency is high. On the contrary, high $V_{\max }$ halos grow slowly at lower redshifts, so that star formation is suppressed by the ionizing background. It is also suggested from these models that more than $70 \%$ of baryon (i.e., $1-F>0.7)$ have been lost from each dwarf satellite, in good agreement with suggestions from chemical evolution models to explain low metallicities of stars in the observed dSphs.

- We have found that the values of $F$ in subhalos calculated from the Illustris simulation are in agreement with those derived from our semi-analytical models, implying that our models may describe the realistic evolutionary histories of dark halos and baryonic matter, although further studies based on much higher-resolution numerical simulations are needed to assess the detailed properties of star formation activities in satellites, especially at small $V_{\max }$.

- The infall rate of gas inferred from the growth rate of dark halos in the common surfacedensity scale shows a peak at the epoch of tidal truncation and this peak epoch is reasonably in agreement with the epoch at which the star formation rate derived for the Milky Way satellites (Fornax and Leo II) is maximum. This also implies that the evolution of a dark halo may play a key role in understanding star formation histories in dwarf satellite galaxies.

We are grateful to the referee for his/her invaluable comments on the manuscript. We also thank Kohei Hayashi for useful discussion, which actually promotes the current work, and Evan Kirby for his constructive comments on an early draft of this paper, which help us improve the manuscript. This work is supported in part by JSPS Grant-in-Aid for Scientific Research (B) (No. 25287062) "Probing the origin of primordial minihalos via gravitational lensing phenomena" and MEXT Grant-in-Aid for Scientific Research on Innovative Areas "Cosmic Acceleration" (No. 15H05889).

\section{REFERENCES}

Boylan-Kolchin, M., Bullock, J. S., Kaplinghat, M. 2011, MNRAS, 415, L40

Boylan-Kolchin, M., Bullock, J. S., Kaplinghat, M. 2012, MNRAS, 422, 1203

Burkert, A. 1995, ApJ, 447, L25 
de Blok, W. J. G., McGaugh, S. S., Bosma, A., \& Rubin, V. C. 2001, ApJ, 552, L23

Di Cintio, A., Brook, C. B., Macciò, A. V., et al. 2014, MNRAS, 437, 415

Donato, F., Gentile, G., Salucci, P., et al. 2009, MNRAS, 397, 1169

Gentile, G., Famaey, B., Zhao, H., \& Salucci, P. 2009, Nature, 461, 627

Gilmore, G., Wilkinson, M. I., Wyse, R. F. G., et al. 2007, ApJ, 663, 948

Gnedin, N. Y. 2000, ApJ, 542, 535

Gnedin, N. Y. 2012, ApJ, 754, 113

Hayashi, K., \& Chiba, M. 2012, ApJ, 755, 145

Hayashi, K., \& Chiba, M. 2015a, ApJ, 803, L11 (HC15a)

Hayashi, K., \& Chiba, M. 2015b, ApJ, 810, 22

Homma, H., Murayama, T., Kobayashi, M. A. R., \& Taniguchi, Y. 2015, ApJ, 799, 230

Ibata, R. A., Lewis, G. F., Conn, A. R., et al. 2013, Nature, 493, 62

Kirby, E. N., Cohen, J. G., Smith, G. H., et al. 2011a, ApJ, 727, 79

Kirby, E. N., Martin, C. L. \& Finlator, K. 2011, ApJ, 742, L25

Kirby, E. N., Cohen, J. G., Guhathakurta, P., et al. 2013, ApJ, 779, 102

Kirby, E. N., Bullock, J. S., Boylan-Kolchin, M., Kaplinghat, M., \& Cohen, J. G. 2014, MNRAS, 439, 1015

Klypin, A., Kravtsov, A. V., Valenzuela, O., \& Prada, F. 1999, ApJ, 522, 82

Kormendy, J., \& Freeman, K. C. 2004, in IAU Symposium, Vol. 220, Dark Matter in Galaxies, ed. S. Ryder, D. Pisano, M. Walker, \& K. Freeman, 377

Kravtsov, A. 2010, Advances in Astronomy, article id. 281913

Kroupa, P., Theis, C., \& Boily, C. M. 2005, A\&A, 431, 517

Lovell, M. R., Eke, V., Frenk, C. S., et al. 2012, MNRAS, 420, 2318

Macciò, A. V., Kang, X., \& Moore, B. 2009, ApJ, 692, L109

Macciò, A. V., \& Fontanot, F. 2010, MNRAS, 404, L16

Mac Low, M.-M., \& Ferrara, A. 1999, ApJ, 513, 142 
Madau, P., Shen, S., \& Governato, F. 2014, ApJ, 789, L17

Martin, C. L., Kobulnicky, H. A. \& Heckman, T. M. 2002, ApJ, 574, 663

Mateo, M., Olszewski, E. W., Pryor, C., Welch, D. L., \& Fischer, P. 1993, AJ, 105, 510

McConnachie, A. W., \& Irwin, M. J. 2006, MNRAS, 365, 902

McQuinn, K. B. W. et al. 2015, ApJ, 815, L17

Milosavljevic̀, M., \& Bromm, V. 2014, MNRAS, 440, 50

Moore, B., 1994, Nature, 370, 629

Moore, B., Ghigna, S., Governato, F., Lake, G., Quinn, T., \& Stadel, J. 1999, ApJ, 524, L19

Navarro, J. F., Frenk, C. S., \& White, S. D. M. 1996, ApJ, 462, 563

Nelson, D., Pillepich, A., Genel, S., et al. 2015, Astronomy and Computing, 13, 12

Oh, S.-H., de Blok, W. J. G., Brinks, E., Walter, F., \& Kennicutt, Jr., R. C. 2011, AJ, 141, 193

Okamoto, T., Gao, L., \& Theuns, T. 2008, MNRAS, 390, 920

Pawlowski, M. S., Pflamm-Altenburg, J., \& Kroupa, P. 2012, MNRAS, 423, 1109

Pawlowski, M. S., Kroupa, P., \& Jerjen, H. 2013, MNRAS, 435, 1928

Pawlowski, M. S., Famaey, B., Merritt, D., \& Kroupa, P. 2015, ApJ, 815, 19

Prada, F., Klypin, A. A., Cuesta, A. J., Betancort-Rijo, J. E., \& Primack, J. 2012, MNRAS, 423, 3018

Salucci, P., Wilkinson, M. I., Walker, M. G., et al. 2012, MNRAS, 420, 2034

Sawala, T., Frenk, C. S., Fattahi, A., et al. 2016, MNRAS, 457, 1931

Simon, J. D., \& Geha, M. 2007, ApJ, 670, 313

Sobacchi, E., \& Mesinger, A. 2013, MNRAS, 432, L51

Strigari, L. E., Bullock, J. S., Kaplinghat, M., et al. 2008, Nature, 454, 1096

Swaters, R. A., Madore, B. F., van den Bosch, F. C., \& Balcells,M. 2003, ApJ, 583, 732

Tegmark, A. et al. 2004, ApJ, 606, 702

Vogelsberger, M., Zavala, J., \& Loeb, A. 2012, MNRAS, 423, 3740

Weisz, D. R., Dolphin , A. E., Skillman, E. D., et al. 2014, ApJ, 789, 147 
Woo, J., Courteau, S., \& Dekel, A. 2008, MNRAS, 390, 1453

Zhu, Q., Marinacci, F., Maji, M., et al. 2016, MNRAS, 458, 1559

This preprint was prepared with the AAS $\mathrm{LAT}_{\mathrm{E}} \mathrm{X}$ macros v5.2. 